\title{
Image Analysis Effects using Image Compression
}

James Grande

\section{GE Global Research Center, Niskayuna, NY 12309}

The extensive adoption of digital imaging and image analysis has resulted in an explosion of digital image storage needs and the use of image compression algorithms in order to reduce the needs of computer storage media. Often, the user simply uses popular compression algorithms such as JPEG and chooses a level of compression that does not diminish the apparent quality to the human eye. The image is then often used to quantify morphological parameters using image analysis tools. In contrast, many users who frequently use image analysis will store digital images in formats that provide no compression (often referred to as "lossless" image reconstruction algorithms). This paper outlines considerations so that users of image analysis tools may tailor use of compression ratios to their types of images so that their storage needs may be greatly reduced while maintaining accurate morphological measurements.

The image analysis program used for this study was the Clemex, Inc. Vision system. The images were saved in various formats and compression ratios using Adobe Photoshop 7. A thermal barrier coating (TBC) image (Figure 1.) was chosen for this study because it contains several types of features that illustrate pertinent considerations. The TBC coating has "low frequency" features (coating) where the average coating thickness and surface roughness are of interest and "high frequency" features (pores) where various morphological parameters such as length, area, and circle diameter are measured.

This study included image compression using two of the most popular compression algorithms: TIFF (Tagged Image File Format) and JPEG (Joint Photographic Experts Group). An additional compression used with these image formats was Lemple-Zif-Welch (LZW) for TIFF format and a quality setting from 0 to 12 for JPEG format.

Compression has very little effect on some of the low-frequency feature measurements such has average coating thickness and roughness (Figures 2 and 3). Compression levels as high as quality setting 3 for JPEG compression can be used with little or no loss of accuracy. This can yield an order of magnitude decrease in storage needs that can be useful if hundreds or thousands of images are routinely stored. However, a parameter such as pore count begins to be significantly affected at a JPEG compression setting of 10.

An example of a good compromise between achieving significant savings in storage space (40\%) while minimizing the effects of reconstructing an image is using the LZW compression option for storing images in TIFF format.

The compression effects chart can be used as a guide to developing best practices in situations that require image analysis while dealing with constraints imposed by computer hardware and support. In addition, factors which include operator ability to distinguish features of interest and image processing algorithms can play a role in deciding which, if any, compression algorithm shall be used. 


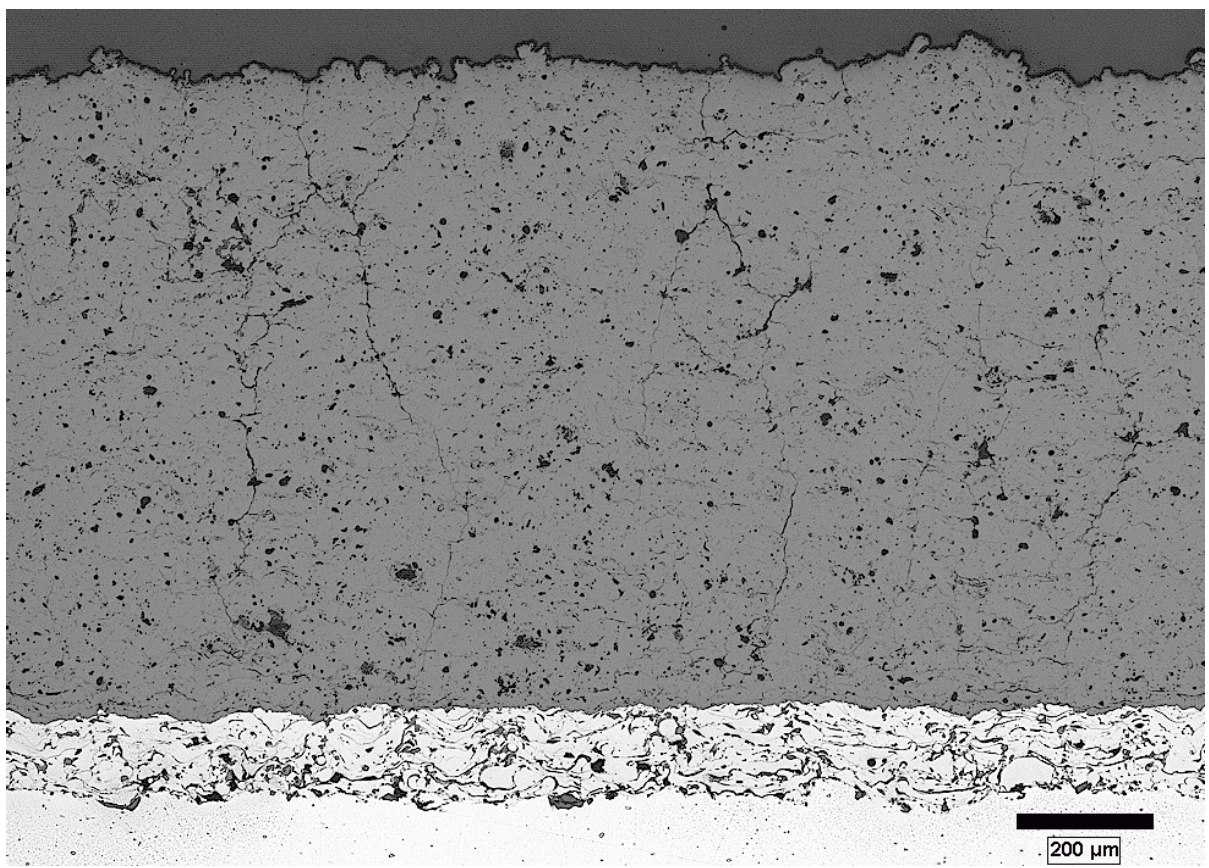

Figure 1.) Thermal barrier coating with features of interest: black pores and gray ceramic coating.

\begin{tabular}{|c|c|c|c|c|c|c|c|c|c|c|c|c|c|}
\hline \multirow{4}{*}{$\begin{array}{r}\text { image format } \\
\text { quality setting/type of compression }\end{array}$} & \multicolumn{13}{|c|}{ IMAGE ANALYSIS COMPARISON CHART } \\
\hline & \multirow{2}{*}{\multicolumn{7}{|c|}{$\begin{array}{l}\text { Type of Image } \\
\text { JPEG }\end{array}$}} & \multicolumn{6}{|c|}{ Difference from Original Digitized Image } \\
\hline & & & & & & & & TIFF & & & JPEG & & \\
\hline & None & $L Z W$ & 12 & 10 & 9 & 6 & 3 & $L Z W$ & 12 & 10 & 9 & 6 & 3 \\
\hline Image Size (kb) & 986 & 592 & 490 & 278 & 186 & 123 & 70 & $-40.0 \%$ & $-50.3 \%$ & $-71.8 \%$ & $-81.1 \%$ & $-87.5 \%$ & $-92.9 \%$ \\
\hline average thickness $(\mu \mathrm{m})$ & 944 & 944 & 944 & 944 & 944 & 945 & 944 & $0.0 \%$ & $0.0 \%$ & $0.0 \%$ & $0.0 \%$ & $0.1 \%$ & $0.0 \%$ \\
\hline tortuosity (L/Lo) & 1.22 & 1.22 & 1.22 & 1.22 & 1.21 & 1.25 & 1.22 & $0.0 \%$ & $0.3 \%$ & $0.1 \%$ & $-0.7 \%$ & $2.5 \%$ & $-0.4 \%$ \\
\hline roughness $(\mu \mathrm{m})$ & 12.1 & 12.1 & 12.1 & 12.0 & 12.1 & 12.4 & 12.0 & $0.0 \%$ & $-0.3 \%$ & $-0.6 \%$ & $-0.2 \%$ & $2.3 \%$ & $-0.8 \%$ \\
\hline porosity (\%) & 7.9 & 7.9 & 7.8 & 7.8 & 7.8 & 7.7 & 8.3 & $0.0 \%$ & $-0.1 \%$ & $-0.3 \%$ & $-0.7 \%$ & $-1.6 \%$ & $6.2 \%$ \\
\hline average linear intercept $(\mu \mathrm{m})$ & 5.2 & 5.2 & 5.1 & 5.3 & 5.5 & 5.8 & 6.4 & $0.0 \%$ & $-0.2 \%$ & $3.6 \%$ & $6.4 \%$ & $11.7 \%$ & $23.6 \%$ \\
\hline pore count (\#) & 3864 & 3864 & 3865 & 3568 & 3364 & 3001 & 2614 & $0.0 \%$ & $0.0 \%$ & $-7.7 \%$ & $-12.9 \%$ & $-22.3 \%$ & $-32.3 \%$ \\
\hline Circle Diameter $(\mu \mathrm{m})$ & 4.8 & 4.8 & 4.8 & 5.0 & 5.2 & 5.5 & 6.18 & $0.0 \%$ & $-0.3 \%$ & $4.7 \%$ & $8.0 \%$ & $15.3 \%$ & $29.1 \%$ \\
\hline Length $(\mu \mathrm{m})$ & 6.8 & 6.8 & 6.8 & 7.1 & 7.4 & 7.9 & 8.92 & $0.0 \%$ & $-0.6 \%$ & $5.2 \%$ & $8.2 \%$ & $15.7 \%$ & $31.3 \%$ \\
\hline Aspect Ratio $(\mu \mathrm{m})$ & 1.60 & 1.60 & 1.60 & 1.63 & 1.63 & 1.65 & 1.70 & $0.0 \%$ & $-0.5 \%$ & $1.8 \%$ & $1.4 \%$ & $2.8 \%$ & $5.9 \%$ \\
\hline & 30.2 & 30.2 & 30.2 & 32.6 & 34.5 & 38.3 & 47.4 & $0.0 \%$ & $-0.1 \%$ & $8.0 \%$ & $14.1 \%$ & $26.8 \%$ & $57.0 \%$ \\
\hline Perimeter $(\mu \mathrm{m})$ & 13.5 & 13.5 & 13.5 & 14.4 & 15.1 & 16.3 & 19.2 & $0.0 \%$ & $0.0 \%$ & $6.8 \%$ & $11.6 \%$ & $21.0 \%$ & $42.2 \%$ \\
\hline
\end{tabular}

Figure 2.) Chart showing data quantifying the effects of compression on various stereological and morphological measurements.

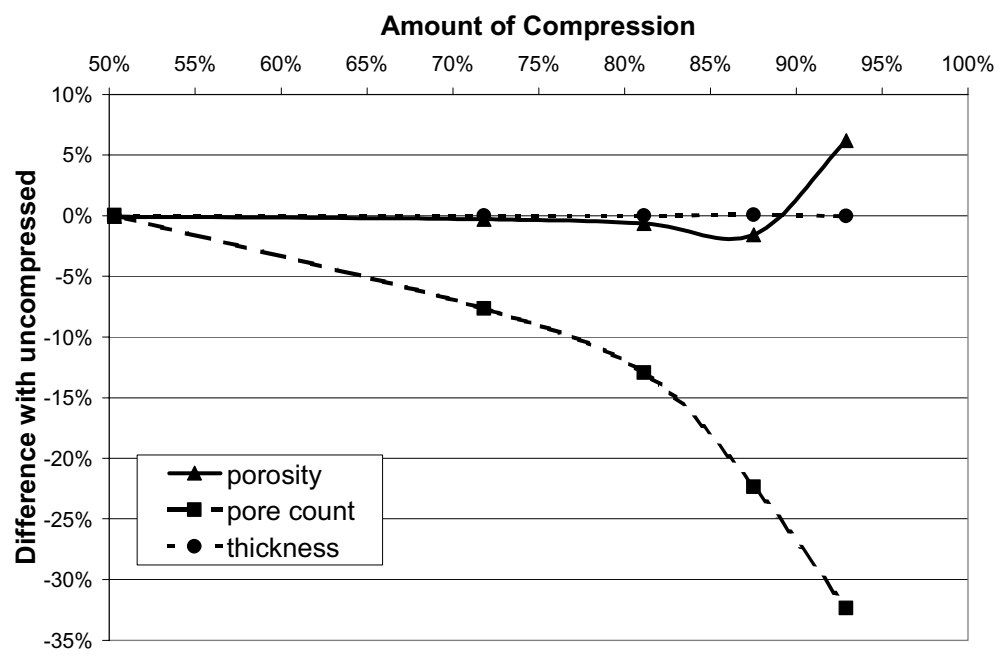

Figure 3.) Compression effects for low frequency (thickness and porosity) and high frequency (pore count) features of interest. 